\title{
Isomerização do óleo essencial de pimenta-longa (Piper hispidinervium C. DC) para a obtenção de isosafrol
}

\author{
Marco Aurelio CREMASCO ${ }^{1}$, Nazareno de Pina BRAGA ${ }^{2}$
}

\section{RESUMO}

As piperáceas brasileiras apresentam propriedades para serem utilizadas, entre outras aplicaçóes, como inseticidas e antifúngicos. Dentre as piperáceas, pode-se citar a Piper hispidinervum C. DC. De suas folhas e dos talos finos extrai-se um óleo essencial rico em safrol, que é utilizado pela indústria como matéria-prima na manufatura, por exemplo, do piperonal. A sequência natural do processo para a obtenção do piperonal é o de submeter o safrol obtido da concentração do óleo essencial à isomerização para seu correspondente isômero estável chamado isosafrol. Apresenta-se neste artigo, por sua vez, a síntese do isosafrol diretamente do óleo essencial de pimenta-longa (Piper hispidinervium C. DC). Este óleo essencial apresenta o safrol como constituinte majoritário, possibilitando a sua isomerizaçáo para a produção de isosafrol, que é empregado nas indústrias farmacêuticas e de fragrâncias. O objetivo deste trabalho é o de apresentar a obtenção do isosafrol sem a necessidade da etapa de separaçáo do safrol do óleo essencial de pimenta-longa. Para tanto, foram realizados ensaios de isomerização do óleo essencial, obtendose uma solução contendo 79,4\% da mistura cis, trans-isosafrol. Ressalte-se que o óleo essencial continha 86,4 \% de safrol, resultando em alto rendimento na sua conversão a cis, trans-isosafrol de $97,1 \%$.

PALAVRAS-CHAVE: Pimenta longa, Óleo essencial; Safrol; Isosafrol; Isomerização.

\section{Isomerization of essential oil of (Piper hispidinervium) C. DC to obtain isosafrole}

\section{ABSTRACT}

The Brazilian peppers present proprieties that can be used in fragrance and pharmaceutical industries, for example. One these pepper is long pepper (Piper hispidinervium C. DC.), who its essential oil is rich in safrole. Safrole is an important compound with application in several industries, such as pharmaceutics, cosmetics. After its chemical conversion, for example, it is possible to obtain heliotropin, a fixative agent for fragrances. The sequence to obtain the heliotropin is by safrole isomerization, that, initially, produce isosafrole. In this work, however, presents the synthesis of isosafrole directly from essential oil of Piper hispidinervium C. DC. In this case, the isomerization of essential oil was done getting 79,4\% of cis, trans-isosafrole mixture. The essential oil presented $86,4 \%$ of safrole, resulting in $97,1 \%$ yield to cis, trans-isosafrole .

KEY WORDS: Piper hispidinervium; Essential oil; Safrole; Isosafrole; Isomerization.

${ }_{1}$ Universidade Estadual de Campinas; Email: cremasco@feq.unicamp.br

2 Email: tobraga@gmail.com 


\section{INTRODUÇÃO}

O Brasil possui tradição no cultivo de plantas aromáticas e na produçấo de óleos essenciais, proporcionando divisas de exportação, sendo crescente a absorção desses produtos por diversos ramos industriais como nos de alimentos, fármacos, perfumes e cosméticos, inseticidas, detergentes e desinfetantes. Isto favorece a pesquisa e o desenvolvimento de novos processos e/ou técnicas de obtenção de tais produtos. Dentre as matérias-primas para a produção de óleos essenciais que despertaram interesse em passado recente, pode-se citar as piperáceas.

As espécies Piper têm sido usadas na medicina tradicional para tratar inúmeras doenças, incluindo males ginecológicos; desordem intestinal; antimicrobial; antioxidante e efeitos citotóxicos (Moreira et al. 1998). Estudos realizados com as piperáceas brasileiras têm mostrado a ocorrência de fenilpropanóides, pironas, lignóides e cromenos, ao lado de amidas que apresentam propriedades para serem utilizadas como inseticidas e antifúngicos (Ciccio e Ballestero 1997; Alécio et al. 1998; Baldoqui et al. 1999; Benavides et al. 1999; Navickiene et al. 2000; Lago et al. 2004).

Entre os fenilpropanóides, a ocorrência do safrol (4-alil1,2-metilenodioxi-benzeno) no óleo essencial de Piper hispidinervium C. DC foi relevante quando se apresentou como uma nova fonte para a síntese da heliotropina (piperonal; 3,4-metilenodioxi-benzaldeído), um fixador de fragrância, e butóxido de piperonila, um inseticida sinergista (Maia et al. 1987).

Pesquisas realizadas resultaram no conhecimento da reatividade do safrol, de tal maneira que todos os átomos de carbono desta substância podem ser eficientes e regiosseletivamente funcionalizados (Barreiro e Fraga, 1999). A isomerizaçáo do safrol para seu correspondente isômero estável, o isosafrol, é uma importante reação de isomerização deste alilbenzeno em que os produtos encontram aplicaçôes nas indústrias de fragrâncias e farmacêuticas. Tradicionalmente tais reaçôes são catalisadas por álcalis, como $\mathrm{KOH}$ em soluçôes alcoólicas em altas temperaturas. A isomeração dessa natureza é comum para conversáo de quase todos os alilbenzenos (como safrol, apiol, dilapiol e elemicina) para o seu correspondente isômero propenilbenzeno, é usualmente executada pelo aquecimento do alilbenzeno junto com um catalisador inorgânico ou mistura de catalisadores sob refluxo, com ou sem uso de um solvente. A isomerização sempre produz uma mistura de isômeros cis-trans do propenilbenzeno (aproximadamente 30:70 usando isomerização padrão catalisada com o íon hidróxido; Bauer, 2002).

No presente trabalho procura-se avaliar a possibilidade da síntese dos isômeros trans-propenilbenzeno (trans-isosafrol) e cis-propenilbenzeno (cis-isosafrol) diretamente do óleo essencial de pimenta-longa (Piper hispidinervium C. DC), partindo do pressuposto que tal óleo essencial é rico em safrol. Esta estratégia mostra-se bastante interessante, sob o aspecto tecnológico, à medida que a etapa de fracionamento do óleo essencial rico em safrol é suprida, resultando na otimização econômica do processo de síntese.

\section{MATERIAIS E MÉTODOS}

O óleo essencial de pimenta-longa utilizado é oriundo de usinas de destilaçáo localizada no Estado do Acre. As amostras foram obtidas pelo método de extração com arraste a vapor das folhas e galhos finos do arbusto. Para as etapas de caracterizaçáo tanto do óleo essencial quanto dos produtos de síntese foram utilizados padróes cromatográficos do safrol (CAS 94-59-7), terpinoleno (CAS 586-62-9), principais componentes do óleo essencial de pimenta-longa; e cis, trans-isosafrol (CAS 120-58-1), principais componentes da isomerização pretendida.

A isomerização do óleo essencial de pimenta-longa a cis, trans-isosafrol foi executada em um baláo de fundo redondo de $250 \mathrm{~mL}$, com condensador de refluxo, posteriormente aquecido e agitado por meio de uma manta aquecedora da FISATOM, modelo 52.

Para a caracterização cromatográfica do óleo essencial de pimenta-longa e dos produtos da síntese do isosafrol foram injetados padróes cromatográficos de concentrações conhecidas. Com esta técnica foi possível caracterizar os compostos do óleo essencial e os produtos da síntese.

A análise cromatográfica do óleo essencial foi realizada em um cromatógrafo em fase gasosa da marca HP5890 II, em coluna capilar HP-20M (Carbovax 20M) com $50 \mathrm{~m}$ de comprimento (diâmetro do filme $=0,25 \mu \mathrm{m}$, diâmetro da coluna $=0,25 \mathrm{~mm}$ ). O detector utilizado nesse equipamento é o de ionizaçáo de chama (DIC). O aquecimento foi de 6 ${ }^{\circ} \mathrm{C} . \mathrm{min}^{-1}$, iniciando em $75^{\circ} \mathrm{C}$ e finalizando a $210^{\circ} \mathrm{C}$. O gás de arraste, Hélio, foi injetado em um fluxo de $1 \mathrm{~mL} \cdot \mathrm{min}^{-1}$. O volume de óleo utilizado para análise foi de $0,5 \mu \mathrm{L}$, injetado diretamente. $\mathrm{O}$ tempo de duração da análise para o óleo essencial foi de $28,5 \mathrm{~min}$.

$\mathrm{Na}$ etapa da síntese do isosafrol foi retirada uma amostra da soluçáo obtida para análise cromatográfica. As amostras foram analisadas por cromatografia a gás utilizando-se um sistema Hewllett Packard 5180 A. O método usado foi split-splitless em coluna de sílica fundida HP-5, de $30 \mathrm{~m}$ (diâmetro do filme $=0,25 \mu \mathrm{m}$, diâmetro da coluna $=0,25 \mathrm{~mm}$ ), programaçáo de temperatura de 60 a $240{ }^{\circ} \mathrm{C}$, com aquecimento à razáo de 3 ${ }^{\circ} \mathrm{C} . \mathrm{min}^{-1}$. O gás de arraste utilizado foi o Hélio, regulado para fornecer uma velocidade linear de $3 \mathrm{~cm} . \mathrm{s}^{-1}$ (medidos a 150 $\left.{ }^{\circ} \mathrm{C}\right)$. Para esta técnica, usou-se injeção manual e detector de 
ionização de chama com temperaturas de $200^{\circ} \mathrm{C}$ e $250^{\circ} \mathrm{C}$, respectivamente (Santos et al. 2006).

Foram realizados, dentro de cada ensaio, três experimentos de isomerização do óleo essencial variando-se o tempo de reação em 5, 10 e 15 min. No final de cada reação de isomerização foi coletada uma alíquota para posterior determinação, por cromatografia gasosa, da concentração obtida em cada ensaio da reação de isomerização. As condiçóes operacionais foram: temperatura de aquecimento da solução de $120^{\circ} \mathrm{C}, 120 \mathrm{~g}$ de óleo essencial e $1 \mathrm{~g}$ de $\mathrm{NaOH}$.

\section{RESULTADOS E DISCUSSÃO}

Os resultados relativos à caracterização cromatográfica do óleo essencial de pimenta-longa estão apresentados na Tabela 1. Constam, nesta tabela, a concentração de cada componente na segunda coluna, assim como o seu teor, em $\%$, na terceira coluna.

Como pode ser observado da inspeção da Tabela 1 , a composiçáo química dos outros compostos corresponde a $11,4 \%$ dos compostos presentes no óleo essencial de pimentalonga. Na composição dos "outros”, Costa (1999) apresenta uma análise cromatográfica em que constam os seguintes compostos: $\alpha$-pineno, mirceno, decano, 3-careno, $p$-cimeno, limoneno, cis- $\beta$-ocimeno, trans- $\beta$-ocimeno, $\gamma$-terpineno, terpinoleno, safrol, metil eugenol, $\alpha$-humuleno, germacreno $\mathrm{D}$, elemicina e germacreno $\mathrm{B}$.

A isomerizaçáo do óleo essencial de pimenta-longa, que pode ser ilustrada na Figura 1, cuja cinética de isomerização está apresentada Figura 2, resultou na formação dos compostos apresentados na Tabela 3, os quais foram identificados mediante cromatografia gasosa.

Comparando-se as Tabelas 1 e 2 verifica-se o aumento da concentraçáo do terpinoleno. Isto pode ter sido causado pela isomerizaçáo de compostos presentes no óleo essencial, como o d-limoneno e $\gamma$-terpineno. Nesta etapa obteve-se uma conversão de $97,1 \%$ do safrol para cis, trans-isosafrol.

Verifica-se da Tabela 2 e da Figura 2 que o composto transisosafrol é formado em maior quantidade, indicando ser este o isômero termodinamicamente mais estável. Observa-se que a isomerização produziu uma mistura de isômeros cis-trans do propenilbenzeno (aproximadamente 25:75).

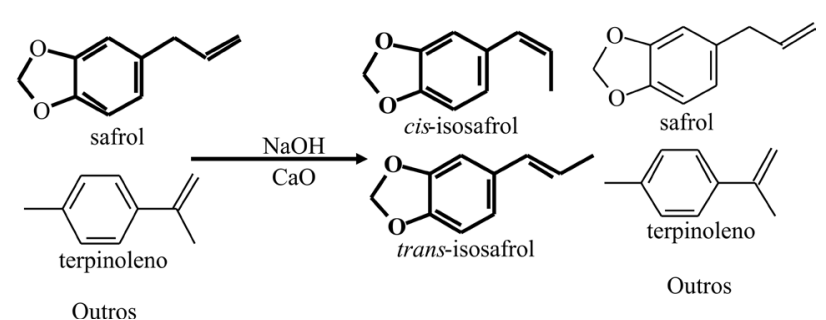

Figura 1 - Isomerização do óleo essencial de pimenta-longa.

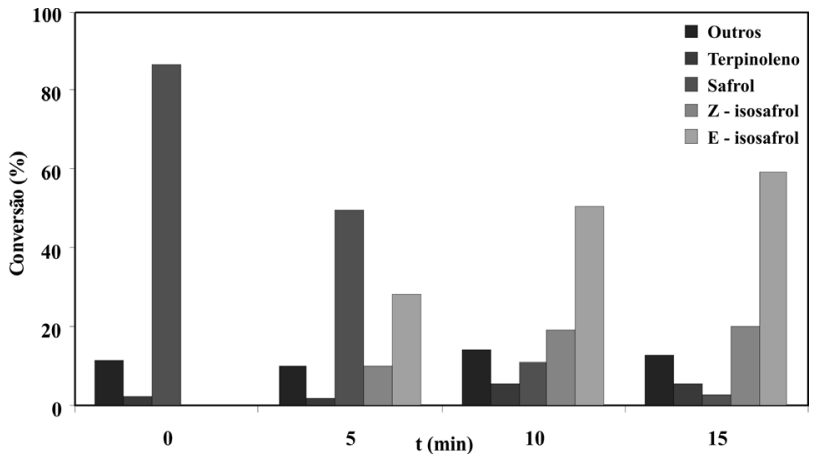

Figura 2 - Cinética da isomerização do óleo essencial a cis, trans-isosafrol.

Tabela 1 - Composição química média do óleo essencial de pimenta-longa.

\begin{tabular}{lcc}
\hline Compostos & $\mathrm{c}\left({\left.\mathrm{mg} . \mathrm{mL}^{-1}\right)}\right.$ & $\%$ no óleo essencial \\
\hline Terpinoleno & $0,008 \times 10^{-2}$ & $2,2 \%$ \\
Safrol & $2,342 \times 10^{-2}$ & $86,4 \%$ \\
Outros & - & $11,4 \%$ \\
\hline
\end{tabular}

Tabela 2 - Composição química média dos produtos de síntese de isomerização do óleo essencial de pimenta-longa.

\begin{tabular}{lcc}
\hline Compostos & $\mathrm{c}\left({\left.\mathrm{mg} . \mathrm{mL}^{-1}\right)}\right.$ & $\%$ no sorbato \\
\hline Terpinoleno & $0,209 \times 10^{-2}$ & 5,4 \\
Safrol & $0,771 \times 10^{-2}$ & 2,7 \\
cis-isosafrol & $0,495 \times 10^{-2}$ & 20,1 \\
trans-isosafrol & $1,238 \times 10^{-2}$ & 59,2 \\
Outros & - & 12,6 \\
\hline
\end{tabular}

\section{CONCLUSÃO}

O presente trabalho relata a obtenção de isômeros de isosafrol a partir da síntese direta do óleo essencial de pimentalonga, sem a necessidade da separaçáo prévia do safrol. Por meio da metodologia empregada obteve-se uma solução contendo 79,4\% da mistura cis, trans-isosafrol. Ressalte-se que o óleo essencial continha $86,4 \%$, no que resulta em alto rendimento na conversão do safrol a cis, trans-isosafrol $(97,1 \%)$.

É importante mencionar que mesmo partindo diretamente de safrol, não se obteria isômeros puros, necessitando, por sua vez, de uma etapa de purificação para a remoção do safrol residual. Esta etapa continua sendo necessária na presente situação, pois além do safrol, existem outras espécies decorrentes da isomerização do óleo essencial, como o terpinoleno. Por outro lado, dado a alta conversão do safrol aos seus isômeros, constatou-se uma viabilidade tecnológica interessante, tendo em vista a possibilidade da supressão do fracionamento do óleo essencial para a obtenção de safrol mais puro, mesmo porque existe a necessidade de separá-lo posteriormente, independentemente se a isomerização dá-se diretamente do safrol ou, do como proposto neste artigo, do óleo essencial de pimenta-longa. 


\section{BIBLIOGRAFIA CITADA}

Alécio, A. C.; Bolzani, V. S.; Young, M. C. M.; Kato, M. J.; Furlan, M. 1998. Antifungal amide from leaves of Piper hispidum. Journal of Natural Products, 61: 637-639.

Baldoqui, D. C.; Kato, M. J.; Cavalheiro, A. J.; Bolzani, V . S.; Young, M. C. M.; Furlan, M.; 1999. New chromene and prenylated benzoic acid from Piper aduncum. Phytochemistry, 51: 899- 902.

Barreiro, E. J. e Fraga, C. A. M. 1999. The utilization of safrole, principal chemical constituent of sassafras oil, in the synthesis of compounds actives in the arachidonic acid cascade: antiinflammatory, analgesic and antithrombotic. Quimica Nova, 22(5): $744-759$.

Benevides, P. J. C.; Sartorelli, P.; Kato, M. J. 1999. Phenylpropanoids e neolignanas from Piper regnellii. Phytochemistry, 52:339 - 343.

Bauer, K.; Garbe, D.; Surberg, H. 2002. Flavors and fragrances, Ullmann Encyclopedia of industrial Chemistry, Electronic Release, $6^{\text {th }}$, Ed..

Ciccio, J. F. e Ballestero, C. M. 1997. Volatile components of Piper aduncum (Piperaceae) leaves and spikes from Costa Rica. Revista de Biologia Tropical, 45: 783 -790.

Costa, C. M. L. 1999. Influence of drying condition on yield and composition of long pepper (Piper hispidinervium C. DC.) essential oil. Dissertação de Mestrado, Departamento de Engenharia Química, Universidade Federal do Pará, Belém, Pará. 214 pp (in Portuguese, with abstract in English).
Lago, J. H. G.; Ramos, C. S.; Casanova, D. C. C.; Morandim, A. A.; Bergamo, D. C. B.; Cavalheiro, A. J.; Bolzani, V. S.; Furlan, M.; Guimarães, E. F.; Young, M. C. M.; Kato, M. J. 2004. Benzoic acid derivatives from Piper species and their fungitoxic activity against Cladosporium cladosporioides and C. sphaerospermum. Journal of Natural Product, 67: 1783 - 1788.

Maia, J. G. S.; Silva, M. L.; Luz, A. I. R.; Zoghbi, M. G. B.; Ramos, L. S. 1987. Pipper species from Amazon richies in safrole. Quimica Nova, 10(3): 200 - 204.

Moreira, D. D.; Guimarães, E. F.; Kaplan, M. A. C. 1998. A chromene from Piper aduncum. Phytochemistry, 48: 1075 - 1077.

Navickiene, H. M. D.; Alécio, A. C.; Kato, M. J.; Bolzani, V. S.; Young, M. C. M.; Cavalheiro, A. J.; Furlan, M. 2000. Antifungal amides from Piper hispidum and Piper tuberculatum. Phytochemistry, 55: 621-626.

Santos, A. S.; Pereira Jr., N.; Silva, I. M.; Sarquis, M. I. M.; Antunes, O. A. C. 2004. Peroxidase catalyzed microbiological oxidation of isosafrol into piperonal. Process Biochemistry, 39: 2269 - 2275.

Recebido em 25/03/2010

Aceito em 06/07/2010 